\title{
UNA APROXIMACIÓN GENERAL AL PROBLEMA DE LOS SENTIMIENTOS EN HUSSERL*
}

\author{
JESÚS MIGUEL MARCOS DEL CANO \\ CSIC. Instituto de Filosofía / Universidad Pontificia Comillas
}

\begin{abstract}
RESUMEN: La fenomenología ha experimentado en los últimos años un creciente interés por las dimensiones afectivas de la conciencia, lo que se podría extender a otros campos del conocimiento (biología, psicología, ciencias sociales...) en lo que se ha conocido como el "giro emotivo». Pero el análisis de sentimientos, afecciones y deseos no es en ningún caso territorio ignoto para la fenomenología: la temática estaba muy viva desde los inicios del movimiento (solo hay que pensar en Scheler) y fundamentó la obra de herederos de Husserl como Heidegger o Henry. Menos conocido es que el propio fundador de la fenomenología se ocupó intensamente con la temática. Ahora que se van a publicar los manuscritos inéditos de Husserl sobre los sentimientos, este artículo ofrece una perspectiva aproximativa general sobre dónde y cómo aborda esta cuestión.
\end{abstract}

PALABRAS CLAVE: sentimientos; fenomenología; intencionalidad; temple de ánimo; giro emotivo.

\section{A general approach to the problem of feelings in Husserl}

ABSTRACT: Phenomenology has experienced in recent years a growing interest in the affective dimensions of consciousness, which could be extended to other fields of knowledge (biology, psychology, social sciences ...) in what has been known as the «Emotional turn». However, the analysis of feelings, desires and affections is not in any case an unknown territory for phenomenology: the theme was very much alive from the beginning of the movement (one only has to think of Scheler) and founded the work of Husserl heirs Heidegger and Henry. Less well-known is that the founder of phenomenology was intensely occupied with the subject. Now that Husserl's unpublished manuscripts on feelings are to be published, this article offers a general approximate perspective on where and how he addresses this subject.

KEY WORDS: feelings; phenomenology; intentionality; stimmung; emotional turn.

\section{INTRODUCCIÓN}

Husserl presentó su programa fenomenológico como una tarea infinita que sería llevada a cabo a través de las generaciones por filósofos convertidos en funcionarios de la humanidad. Esta vasta pretensión revela ya muchas particularidades del carácter de su obra filosófica. Una de ellas es su confianza en el trabajo metódico y disciplinado, que él entendía como mucho más fértil que la crítica apresurada. Así, pensaba que lo que él estaba haciendo, llenando miles y miles de manuscritos de investigación, era tan radical y único que cualquiera que quisiera en el futuro pensar las cosas desde su origen tendría que volver a pasar por ellos. Tenía su filosofía la vocación de cimentarse desde

* Este artículo se enmarca dentro de dos proyectos de investigación: «Fenomenología del cuerpo y análisis del dolor II» (FFI2017-82272-P), del Instituto de Filosofía del CSIC y financiado por el Ministerio de Industria, Economía y Competitividad; y «Reconocimiento del otro y justicia (REJUS)», financiado por la Universidad Pontificia Comillas. 
los primeros principios, pero a diferencia de la antigua escolástica, no a partir de la especulación racional, sino desde la descripción minuciosa y analítica de lo experimentado, es decir, de lo vivido. Y dentro de lo vivido no hay razón para dejar fuera nada: están las cosas que se presentan a la conciencia, con estas y aquellas características; están los juicios que emitimos sobre esas cosas, describiendo precisamente sus características y haciendo clasificaciones a partir de ellas; están por supuesto los otros, cuya presencia constituye uno de los «objetos» de estudio más decisivos en fenomenología. Pero no se puede prescindir de otro ámbito, a veces relevado a categoría secundaria en filosofía, que englobaría toda una serie de fenómenos de importancia excepcional para la vida: se trata de la región afectiva, en la que los sentimientos juegan un rol central.

Ya Franz Brentano había elevado el sentimiento a una de las tres categorías fundamentales de los fenómenos psíquicos, junto a las representaciones y los juicios, en lo que denominaba "actos de amor y odio», género que también incluía los fenómenos de la voluntad ${ }^{1}$. De hecho, la discusión sobre los sentimientos, su naturaleza, su forma de manifestarse, su relación con otros fenómenos de la conciencia y con los objetos, su legitimidad en el ámbito de lo ético e incluso su valor epistemológico, estaba muy viva en la filosofía y psicología de finales del XIX y principios del XX, la época de la irrupción de Husserl. Nombres como Alexius Meinong, Theodor Lipps, Carl Stumpf (director del trabajo de habilitación universitaria de Husserl), el propio Franz Brentano, Alexander Pfänder, Moritz Geiger y especialmente Max Scheler, dedicaron monografías y artículos de extraordinaria riqueza y radicalidad a la cuestión afectiva. Husserl, que estaba muy al tanto de todo ello, participó activamente en estas discusiones. Pese a que la temática quedó un tanto relegada en su obra publicada en vida (como tantas otras), una mirada retrospectiva a través de textos de sus obras completas (Husserliana) y de manuscritos de investigación de inminente publicación revela que el filósofo se ocupó a fondo con la cuestión.

Su interés se redobla al afectar directamente a núcleos teóricos y conceptos fundamentales del método fenomenológico. Es el caso de la intencionalidad o de la teoría de los actos en la quinta de las Investigaciones lógicas. Si Husserl llega a preguntarse por los sentimientos es precisamente porque este fenómeno presenta una serie de rasgos peculiares que cuestionan las descripciones esenciales de la conciencia, fundadas en la clave de bóveda que es la intencionalidad. La posterior división de las vivencias intencionales en actos objetivantes y no-objetivantes, que viene a sustituir a la comentada tríada brentaniana, también va a estar fuertemente determinada por las vivencias afectivas y volitivas: los sentimientos representan con luz meridiana, aunque sin completarla, la clase de actos no-objetivantes. Otro problema que afecta de forma radical a la

1 Brentano, F., Psicología. Traducción de José Gaos. Madrid: Revista de Occidente, 1935, p. $83-96$. 
fenomenología es si tenemos un acceso originario a los sentimientos desde la descripción reflexiva, motivo señalado audazmente por Moritz Geiger (como se verá al final de este artículo), lo que adelantará futuras enmiendas a la fenomenología trascendental y que Husserl se esforzó en contestar. Naturalmente, gran parte del trabajo de Husserl sobre los sentimientos va a estar orientado al ámbito de la ética. Sus numerosos intentos de establecer analogías entre la conciencia objetivadora representativa, por un lado, y por el otro la conciencia afectiva, que presenta objetos como valores que se manifiestan en el sentimiento, buscaban sobre todo fundamentar una axiología formal a la manera de la lógica formal (intento que encontrará no pocos obstáculos). Esta vinculación de la esfera afectiva con la reflexión ética se hará mucho más notoria en la comprensión que Husserl ofrece del sentimiento en los libros de Ideas y está relacionada con los exhaustivos análisis que realizó sobre el tema entre los años 1909 y 1911.

Estos dos años van a ser excepcionalmente fecundos en la exploración fenomenológica de lo afectivo. En ningún otro momento Husserl se empleó tan a fondo con una cuestión que había trabajado desde bastante antes de Investigaciones lógicas. A esta época pertenecen sus innovadores análisis de los temples de ánimo (Stimmungen), a los que Husserl concederá un estatus propio en la estructuración de la esfera sentimental y que, siguiendo con las analogías, vendrían a desempeñar el rol, en el plano del sentimiento, que juega el denominado "horizonte» en el que por necesidad se ofrecen las cosas en la percepción visual. Sin embargo, sus primeros escritos conocidos sobre la esfera afectiva se remontan a los años que van de 1890 a $1894^{2}$, cuando describía el sentimiento como un impulso dinámico y lo vinculaba a la vivencia del interés, propiciando la pregunta de si se trataba de un acto o un estado. También antes de 1900 existen estudios (solo conocidos a través de manuscritos) sobre los movimientos afectivos en los actos de aprobación, que fueron recuperados por Husserl como material de trabajo entre los años 1907 y 1908. Las lecciones de ética de 1902 y del curso 1908/09 presentan, respectivamente, críticas a la comprensión de los sentimientos de Hume y $\mathrm{Kant}^{3}$ y estudios analíticos que aportan más luz a su perseguida conexión entre el pensar, el juzgar y el valorar $^{4}$. Sin versar propiamente acerca del fenómeno del sentimiento, tienen especial relevancia para esta temática los análisis sobre el instinto en la época de su llegada a Friburgo (1916-1918), coincidente con los años de la Primera Guerra Mundial. También aquí aborda el tema del cumplimiento instintivo, así como el estudio de las acciones que se dejan dirigir por el instinto. La relación del instinto con las esferas de la actividad y la pasividad y su vinculación

2 Husserl, E., Aufsätze und Rezensionen (1890-1910) (Husserliana XXII). The Hague: Martinus Nijhoff, 1979, pp. 411-413. (Citado como Hua XXII).

3 Husserl, E., Vorlesungen über Ethik und Wertlehre (1908-1914) (Husserliana XXVIII). Dordrecht: Kluwer, 1988, pp. 384-417. (Citado como Hua XXVIII).

4 Husserl, E., Hua XXVIII, «Die Unterscheidung und das Verhältnis zwischen theoretischer und axiologischer Vernunft», pp. 237-340. 
al «yo puedo» y al problema de la libertad de la voluntad también son señalados. Asimismo, en la época previa a su jubilación, años 1926 y 1927, y en el marco de sus investigaciones sobre la razón práctica y las relaciones de la razón con la ciencia, la moral y la metafísica, Husserl se va a ocupar con el tema del amor personal y el amor al prójimo 5 .

Antes de exponer algunos núcleos de discusión de esta trayectoria ${ }^{6}$, prestando especial atención naturalmente a lo dicho sobre el tema en Investigaciones lógicas y los libros de Ideas, merece la pena destacar algunos aspectos adicionales acerca de la exploración husserliana de los sentimientos. En primer lugar, insistir en que la problemática de los sentimientos no es una cuestión menor en el campo de trabajo fenomenológico. Como buen discípulo de Brentano, Husserl era muy consciente de que la cientificidad de la filosofía tenía su fundamento en la descripción de las estructuras de la conciencia y sus fenómenos, descripción que hay que entender no como una descripción empírica de hechos de conciencia, sino como la mostración esencial de dichas estructuras y fenómenos, es decir, tal y como necesariamente se ofrecen en la experiencia. En su estudio totalizador de la conciencia, la región afectiva, y más en concreto la referida a los sentimientos, fue ganando legitimidad para Husserl y ocupando una posición cada vez más prominente. En este sentido, hay que destacar que el sentimiento cuenta con un cierto privilegio si se quiere abordar las esferas de la receptividad y la pasividad, así como todo lo que tiene que ver con los estratos originarios de la conciencia y con las dimensiones genéticas de la experiencia, temáticas todas ellas por las que Husserl fue mostrando una preocupación creciente. En segundo lugar, reconocer la especial dificultad del objeto de análisis, el sentimiento, que por momentos fuerza y somete a tensión doctrinas y conceptos esenciales de la fenomenología, llegando a cuestionar si no sería necesaria su revisión, lo que en principio no tiene que presentarse como algo negativo. Porque, en tercer lugar, en los análisis del sentimiento de Husserl ya se intuye la fecundidad y riqueza de futuros desarrollos del pensamiento fenomenológico, muchos de los cuales bebieron directamente de la fuente del maestro de Friburgo ${ }^{7}$.

\footnotetext{
5 Todos estos temas se pueden encontrar en: HusserL, E., Grenzprobleme der Phänomenologie. Analysen des Unbewusstseins und der Instinkte. Metaphysik. Späte Ethik. Texte aus dem Nachlass (1908-1937) (Husserliana XLII). Dordrecht: Springer, 2014.

6 El esfuerzo de mayor alcance por recopilar críticamente el conjunto las investigaciones husserlianas sobre los sentimientos se puede encontrar en: Melle, U., «Husserls deskriptive Erforschung der Gefühlserlebnisse», en Life, Subjectivity \& Art: Essays in Honor of Rudolf Bernet, Phaenomenologica 201, Breeur, R. y Melle, U. (eds.), Dordrecht: Springer, 2012, pp. 51-99.

7 El surcoreano Nam-In Lee deja entrever la influencia que pudieron tener sobre Martin Heidegger los escritos de Husserl sobre el sentimiento: LEE, N.-I., «Edmund Husserl's Phenomenology of Mood», en Alterity and Facticity: New perspectives on Husserl, N. Depraz \& D. ZaHAVI (eds.), Drodrecht: Kluwer, 1998, pp. 103-120.
} 


\section{Sentimientos E INTENCIONALIDAD hasta INVESTIGACIONES LÓGICAS}

En Investigaciones lógicas Husserl corrige la certeza brentaniana de que todo contenido de conciencia es intencional. La corrección afecta a los datos de sensación y a algunos sentimientos. Al análisis de lo que pasa con los sentimientos dedica el famoso parágrafo 15 de la Quinta investigación, donde divide esta clase de fenómenos según sean intencionales o no. Pero esta no era la primera vez en que Husserl examinaba la intencionalidad a la luz del sentimiento. En la última década del XIX, Husserl se ocupó de los análisis del cumplimiento intuitivo en el suceso del conocimiento, donde los sentimientos juegan un papel de especial relevancia. En su comentario crítico al artículo de Carl Stumpf sobre La psicología del sonido, en el que este último conectaba el fenómeno de la atención y el interés con el placer (Lust am Bemerken), Husserl se fija en un tipo muy determinado de sentimientos, como por ejemplo la carencia (Mangel) o la satisfacción (Befriedigung), que vendrían a subrayar afectivamente y de algún modo a orientar y dirigir el transcurso del acceso intuitivo a las cosas. Se trata de impulsos del sentimiento que operan en la pareja consecutiva de tensión/resolución presente en todo acto de conocimiento.

«En la transición de la tensión a la resolución sucede el conocimiento. (...) En ese sentido, la tensión está referida al conocimiento. Ella está dirigida al conocimiento. Y también es causa del conocimiento. El conocimiento es, por lo tanto, un efecto elemental del interés en tensión. El interés resuelto es conocimiento en sí mismo.» ${ }^{8}$

Esta idea ya aparecía en el texto fechado entre 1890 y $1894^{9}$ y mencionado anteriormente: Husserl describía el interés como un tipo de sentimiento que acompaña por debajo cada acto intencional. El sentimiento sería esa fuerza dinámica que acompaña todo acto y lo dirige hacia su objeto, lo que indica que forma parte esencial del conocimiento. La intencionalidad misma era comprendida en estas tempranas reflexiones en términos de interés, como un acto mental que incluye un componente afectivo. Un acto se refiere a un objeto, es decir, es intencional si ese objeto despierta nuestro interés.

En el artículo crítico con Stumpf, la comprensión del interés va a abrir la discusión de si los sentimientos son actos o son estados, es decir, si tienen o

8 Husserl, E., Wahrnehmung und Aufmerksamkeit. Texte aus dem Nachlass (1893-1912). Husserliana XXXVIII. Dordrecht: Springer, 2004. p. 159. (Citado como Hua XXXVIII). (La traducción es mía: «Im Übergang von Spannung zu Lösung tritt Erkenntnis ein. (...) Insofern hat die Spannung Beziehung zu Erkenntnis. Sie ist gerichtet auf Erkenntnis. Und sie ist auch Ursache der Erkenntnis. Erkenntnis ist also primäre Wirkung des gespannten Interesses. Das gelöste Interesse ist Erkenntnis selbst»).

9 Husserl, E., Hua XXII, pp. 411-413. Se trata de un apéndice que lleva por subtítulo: Zur Wurzel des Begriffspaars Intention-Erfüllung, que traducido quiere decir «En la raíz de la pareja conceptual intención-cumplimiento». Lo que está en la raíz es, precisamente, ese sentimiento de interés, lo que muestra que Husserl identificaba el lugar del sentimiento en la base de la intencionalidad. 
no intencionalidad: el interés parece indudablemente un acto, ya que manifiesta una actividad dirigida a un objeto, pero no así el placer (ni curiosamente tampoco la alegría ${ }^{10}$, en oposición a lo que más tarde establecerá en Investigaciones Lógicas), que se comporta de forma pasiva y receptiva. Esto va a llevar a Husserl a un bosquejo de clasificación de comportamientos afectivos en los que la intencionalidad está manifiestamente presente ${ }^{11}$, a partir de la que va a considerar el interés como un fenómeno de la voluntad emparentado con el afán y distinto del placer, que es solo un estado. Estas investigaciones sobre suelo vacilante no ocultan su carácter de provisionalidad y son la antesala del abordaje más concluyente que se realiza en Investigaciones lógicas.

En su obra de 1901 Husserl va a practicar una escisión más decidida en el terreno de los sentimientos entre aquellos que considera intencionales (agrado o desagrado, alegría o tristeza) y los no intencionales (los denominados sentimientos sensibles como el placer, el displacer o el dolor). Los primeros presentarían una inequívoca referencia a un objeto, como por ejemplo el agrado por una melodía, mientras que los segundos serían meros estados que considerados en sí mismos no remiten más allá de sí. Este «considerados en sí mismos» es fundamental, ya que los sentimientos intencionales son impensables sin su referencia al objeto, como ocurre por ejemplo en el caso de una convicción, un deseo o una aprobación (esta última será tematizada ampliamente en manuscritos de los años posteriores). Por su parte, la conciencia de sentimientos sensibles como el dolor parece volverse sobre sí misma, sin apuntar esencialmente al objeto. Es cierto que podemos relacionar estos sentimientos con algo objetivo (Husserl pone el ejemplo del dolor de quemarse y el objeto que quema), pero lo hacemos en base a un criterio exterior, no de la misma manera que en el caso de una convicción, que lleva en sí misma una inseparable referencia a algo distinto de ella misma. Se trata, afirma Husserl, de fenómenos de distinto género, aunque se identifiquen con el mismo nombre de sentimientos. Y en este momento se desarrolla una comprensión que va a estar en el centro de las reflexiones husserlianas sobre los sentimientos: su analogía con la esfera teórica-judicativa.

"Se suscita la duda acerca de si estos dos tipos de "sentimientos" pertenecen efectivamente al mismo género único. Antes hablamos de "sentimientos" de gusto o disgusto, aprobación o desaprobación, estima y repudio, vivencias todas que están evidentemente emparentadas con los actos teóricos del asentir y disentir, de considerar probable e improbable, o con los actos de tomar una decisión judicativa o volitiva después de haber sopesado las cosas, etc. No se puede clasificar dentro de la evidente unidad de este género — que abarca

10 Husserl, E., Hua XXXVIII, p. 175: «Pero la alegría no está dirigida a lo que alegra, sino que llena mi alma y solo se refiere a lo que alegra en la reflexión, en la que aparece como razón de la alegría» (La traducción es mía: «Aber Freude ist nicht auf das Erfreunde gerichtet, sondern füllt meine Seele aus und wird erst durch Reflexion auf das Efreunde bezogen, in dem dieses als Grund der Freude erscheint»).

11 Ibid., 178. 
en exclusiva actos- las sensaciones de dolor y placer; más bien pertenecen, desde el punto de vista descriptivo, al mismo género que las sensaciones del tacto, el sabor, el olfato, etc. En el hecho de que a lo sumo sean contenidos presentantes o quizá objetos de intenciones, pero no intenciones ellas mismas, se hace patente una diferencia descriptiva tan esencial que no podemos pensar seriamente en mantener la unidad de un auténtico género. Cierto que en los dos casos, tanto para los actos del gusto que arriba mencionamos, como para estas sensaciones que ahora hallamos, se habla por igual de "sentimientos"; pero que así suceda no puede constituir para nosotros un problema, de la misma manera que no nos dejamos engañar por que suela hablarse de sentir, en la acepción de tocar, a propósito de las sensaciones táctiles. ${ }^{12}$

Los sentimientos intencionales van a ser vivencias que muestran una proximidad a los actos teóricos-objetivantes, en concreto al juicio, ya que parecen expresar una cierta toma de posición (agrado o desagrado, aprobación o desaprobación, amar u odiar...). Por su parte, los fenómenos de la conciencia que conocemos como placer o dolor se sitúan más cerca de las sensaciones del tacto o el sabor, «en el hecho de que a lo sumo sean contenidos presentantes o quizá objetos de intenciones, pero no intenciones ellas mismas». Estos sentimientos sensibles desempeñarían en la estructura de los sentimientos intencionales un rol análogo al de las sensaciones visuales en los actos perceptivos: las sensaciones visuales, como momentos ingredientes de las representaciones, no aparecen propiamente, sino que son utilizadas en la aprehensión para que el objeto aparezca. De la misma manera se comportarían el placer y el dolor en los actos del sentimiento del agrado o la alegría por algo. Serían, como las llama Husserl, sensaciones del sentimiento (Gefühlsempfindungen), frente a los sentimientos propiamente intencionales.

Pero todavía hay otro núcleo teórico en Investigaciones lógicas que afecta directamente a los sentimientos (y a la vida afectiva en general) y que también procede de una reformulación de tesis previas de Brentano, en concreto, de su teoría de los actos. Como ya se ha señalado, Brentano dividió las vivencias intencionales en tres tipos: representaciones, juicios y actos de amor y odio (donde incluía sentimientos y voluntad). Husserl va a criticar este modelo en el capítulo tres de la Quinta investigación lógica, negándole el privilegio único de acto básico a la mera representación y unificándola con los juicios en la categoría fundante de actos objetivantes. Del otro lado, principalmente los sentimientos y las vivencias de la voluntad y el deseo caerán dentro de lo que denominó actos no-objetivantes. Aquí habría que precisar que algunos actos del entendimiento, como por ejemplo el acto de preguntar, que parecerían pertenecer al primer grupo, se incluyen por el contrario dentro del segundo, por lo que no hay una coincidencia completa entre actos del entendimiento y los actos objetivantes, por un lado, y actos del sentimiento y la voluntad y actos no-objetivantes, por el otro.

12 Husserl, E., Logische Untersuchungen (Husserliana XIX/2), The Hague: Martinus Nijhoff, 1984. (Citada como Hua XIX/2), p. 407. (Traducción inédita de Miguel García-Baró). 
¿Pero cuál es el criterio unificador de estos actos objetivantes? Que el acto pueda ser ponente o no-ponente, es decir, que pueda tomar partido por el ser de su objeto, característica que solo concierne a las representaciones y que no incumbe a la clase de los sentimientos. Desde aquí, Husserl va a señalar al término «representación» en el lema brentaniano de que «todo acto intencional o es una representación o tiene una representación a su base» y lo va a sustituir por «acto objetivante». Los sentimientos, por tanto, estarían siempre construidos sobre un acto objetivante. No tendrían un objeto propio, sino que lo tomarían prestado de una representación subyacente. Se servirían de la materia intencional de esta última para ganar su propia condición de acto.

\section{La AFECTIVIDAD OBJETIVANTE DE IDEAS}

El esquema va a modificarse radicalmente en Ideas I (1913). Pero en medio, tiene lugar el gran período de investigación de Husserl sobre la conciencia afectiva, intensificado entre los años 1909 y 1911 y que corre paralelo a algunas de sus más famosas lecciones sobre ética y teoría del valor, recogidas en el volumen 28 de Husserliana ${ }^{13}$. Este período de inmersión en la región de la afectividad, desde múltiples puntos de vista y orientaciones, pondrá las bases de la posición presentada en Ideas I. Conviene aquí indicar que el rendimiento del ingente trabajo realizado por Husserl en estos años queda un tanto adelgazado en las páginas de su obra de 1913. Si bien la perspectiva de Ideas I es innovadora y hasta cierto punto sorprendente, el análisis de lo afectivo y en concreto del sentimiento queda en buena medida ladeado hacia el ámbito de la teoría del valor, privilegiando una identificación del sentimiento con la estimación y dejando en la sombra el ámbito de la pura afección. En las lecciones de ética y de la teoría del valor de esos años (sobre todo 1908/1909 y 1911) se manifiesta el esfuerzo de Husserl por poner los cimientos de una doctrina objetivadora de lo afectivo en la que traza continuas analogías entre esta esfera y la esfera teórica-judicativa. En todo momento hay un interés orientado a la ampliación de la racionalidad al ámbito del sentimiento que tendría su fundamento en la estructura intencional de estos últimos, entendida esta como una «mención» inicial que busca cumplimiento desde su enraizamiento en una posición de creencia. Así parece confirmarlo este texto extraído de sus lecciones de ética y que nos sirve de antesala a nuestra incursión en Ideas I:

«En consideración a la claridad, advierto que la palabra razón (Vernunft) no está entendida aquí en el sentido de una facultad del alma humana, esto es, en el sentido psicológico habitual, sino que comprende un nombre para la clase esencialmente cerrada de actos y sus correspondientes correlatos de actos que están bajo la idea de legalidad e ilegalidad, o de verdad y falsedad,

13 Un estudio a fondo en español sobre estos temas y revisado recientemente en una segunda edición ampliada está recogido en: Ferrer, U. y Sánchez-Migallón, S., La ética de Edmund Husserl, Sevilla: Thémata, 2018. 
de existencia y no-existencia. Así, cuantos tipos diferentes de actos podamos distinguir para los cuales valga esto, tantos tipos de razón. (...) La razón lógica tiene la singular ventaja de que no solo en su propio campo, sino en el campo de todos los demás géneros de menciones - por tanto, en todas las otras esferas de la razón-, formula corrección, determina la legalidad, predica y expresa leyes de corrección. Las razones valorativa y práctica son, por así decir, mudas y en cierto modo ciegas. ${ }^{14}$

Mudas y ciegas, pero bajo el dominio de una idea de legalidad orientada a un cumplimiento. El esfuerzo objetivador de la región del sentimiento culmina en Ideas I. Los actos afectivos y volitivos (donde se incluyen vivencias de agrado y desagrado, del valorar, del desear, revolverse, actuar...) se siguen considerando vivencias intencionales de nivel superior fundadas en representaciones (percepciones, recuerdos, también los juicios, etc.). Esta estratificación expresa que la vivencia de nivel superior puede cesar sin que la vivencia restante pierda su condición íntegra de acto. Una primera diferencia respecto a Investigaciones lógicas es que la nóesis afectiva (en el caso en el que estamos, el sentir) va a presentar un correlato noemático propio, en lugar de tomar prestada la materia del acto fundante, como ocurría en el anterior modelo. No puede haber un momento noético sin su momento noemático que le corresponda específicamente. Y este momento noemático no se limita únicamente a añadir un carácter noemático al nóema fundante de la representación, sino que constituye un objeto propio:

«Toda agregación de caracteres noéticos o toda modificación de los antiguos, no sólo constituye nuevos caracteres noemáticos, sino que eo ipso se constituyen para la conciencia nuevos objetos existentes; a los caracteres noemáticos corresponden caracteres predicables en el objeto del sentido, como predicables reales y no tan solo modificados noemáticamente. ${ }^{15}$

¿Dónde encuentra Husserl ese objeto en el caso del sentimiento? En el valor. El sentimiento encuentra belleza o fealdad, bondad o maldad, objetos de valor mismos. Aquí se abre una esfera completamente heterogénea a la representativa: como se ha dicho, no se trata de una determinación parcial de la representación (que no por ello deja de estar a la base como fundante), sino que los valores de las cosas instituyen una nueva dimensión objetiva del sentido. En todo acto de nivel superior como el de una valoración, tendríamos por tanto el objeto que funda la conciencia de valor (es decir, el objeto en sus determinaciones meramente representativas), el objeto de valor mismo (el valor concreto mismo o la objetidad ${ }^{16}$ del valor, eso que añade la valiosidad al objeto) y finalmente el todo formado por el objeto y el valor (objeto valioso).

14 Husserl, E., Hua XXVIII, p. 68. (Traducción extraída de Ferrer, U. y Sánchez-MigaLLÓN S., o. c., pp. 107-108).

15 Husserl, E., Ideas relativas a una fenomenología pura y una filosofía fenomenológica. Trad.: Antonio Zirión Quijano México D.F.: Fondo de Cultura Económica, 2013, p. 338.

16 Con este término se traduce la «Wertobjektität» husserliana, que evitando el neologismo podría traducirse como la «valía del valor» (con el riesgo de perder el énfasis sobre la comprensión objetiva del valor). 
«La objetidad de valor implica su cosa, y aporta como nueva capa objetiva la valiosidad.» ${ }^{17}$

Un segundo alejamiento de Investigaciones lógicas es que esta nueva conciencia es calificada por Husserl como ponente, que otorga al valor el carácter de existente y, por tanto, la posibilidad de caer bajo modalizaciones dóxicas como la certeza, la duda, la suposición, etc.

«En virtud de una legalidad perteneciente una vez más a la esencia fundamental general de la conciencia, todo cogito puede ser llevado a una protoposición dóxica. (...) Hallaremos razones para ensanchar el concepto de tesis hasta abarcar todas las esferas de actos y, así, para hablar, por ejemplo, de tesis de agrado, tesis de deseo, tesis de voluntad, con sus correlatos noemáticos, "grato", "deseado", "prácticamente debido", y similares. También estos correlatos admiten, mediante la conversión posible a priori del acto respectivo en una prototesis dóxica, la forma de modalidades de ser en un sentido sumamente ampliado. ${ }^{18}$

Me agrada lo agradable de una cosa, pero lo agradable se torna dudoso; o supongo a la cosa como agradable, para posteriormente cerciorarme de lo agradable y llegar a la conciencia cierta de que la cosa es agradable. Del carácter ponente del sentir se extrae una consecuencia bastante sorprendente. Antes habíamos señalado que es precisamente la condición de ser ponente o no el criterio de determinación de los actos objetivantes, lo que quiere decir que también lo son los actos del sentimiento y la voluntad. Así lo concluye Husserl:

«Tras todo ello, el resultado es que todos los actos en general —incluso los actos afectivos y de la voluntad- son objetivantes, primigeniamente constituyentes de objetos, fuentes necesarias de diversas regiones del ser y por ello también de las ontologías correspondientes.»19

Al ser considerados actos ponentes, los sentimientos «toman posición»o «mientan» afectivamente un objeto, que en su caso es un valor. Como todo objeto, presenta sus caracteres dóxicos y puede ser expresado lógicamente, lo que lleva a Husserl a concluir que todo acto o todo correlato de acto alberga en sí algo lógico, explícita o implícitamente.

Las complicaciones a las que da lugar el modelo de Ideas I son sobresalien$\operatorname{tes}^{20}$, lo que obliga a Husserl a diseccionar profusamente los actos intencionales en su nueva y enmarañada complejidad (tema que puede ser motivo para otro artículo). Cabría extraer, sin embargo, dos apreciaciones en relación a este nuevo esquema. En primer lugar, la cuestión de la relación entre la conciencia representativa - la propia del acto fundante- y la conciencia afectiva — fun-

\footnotetext{
17 Ibid., p. 313.

18 Ibid., 355-6.

19 Ibid., 368.

20 Un análisis de referencia sobre esta cuestión se encuentra en: SERRANO DE Haro, A., «Actos básicos y actos fundados. Exposición crítica de los primeros análisis husserlianos» en Anuario Filosófico, Vol. 28, Número 1, 1995, pp. 61-89.
} 
dada en la anterior, pero también objetivadora y con su propia doxa-. Como ya se ha señalado, que el acto afectivo se dirija a su propio objeto no es óbice para que pierda su carácter de acto fundado en una representación fundante. Sin embargo, ahora parece presentar una cierta autonomía, no solo en el sentido de que se ha asegurado su propio objeto, sino también porque tiene su propia doxa, independiente de la doxa fundante. Piénsese por ejemplo en el agrado estético, en el que es indiferente la posición de existencia del correlato captado. En segundo lugar, la nueva compresión de la dimensión afectiva, en concreto de los sentimientos, se abre al valor de la verdad. La posesión de su propia doxa - el poner a la cosa como bella- y sus distintas modalizaciones - «es probable que sea bella», «ahora dudo de que sea bella», «conjeturo que es bella»- comporta que este "valorar como acto ponente» que es el sentimiento en Ideas I presente un carácter objetivo de verdad o falsedad.

\section{Los STUdien y EL TEMPLE De ÁNIMO}

La plasmación de los resultados sobre la región del sentimiento en Ideas I es probablemente la mejor síntesis que Husserl pudo realizar tras un esfuerzo investigador que, como se ha señalado, se intensificó en los años previos a su publicación. Este esfuerzo se recoge en su integridad en el volumen número 43 de Husserliana (en estado de pre-publicación en el momento de escritura de este artículo). Se trata de cuatro volúmenes bajo el título de Studien zur Struktur des Bewusstseins, que sacan a la luz una edición de manuscritos realizada a mediados de los años 20 por Ludwig Landgrebe, asistente de Husserl en aquella época. En el archivo Husserl de Friburgo se puede consultar una copia del trabajo realizado por Landgrebe, que ya incluye los títulos de cada volumen y los índices preparatorios de una obra que Husserl, como en tantos otros casos, decidió no publicar (pero que estuvo a disposición de sus colaboradores, entre ellos Martin Heidegger). Uno de los volúmenes está dedicado al tema del «afecto» o «ánimo» (Gemüt) y amplía exponencialmente la panorámica sobre los sentimientos en relación a los mencionados desarrollos de Investigaciones lógicas e Ideas $I$.

Una mirada global a estos textos permite encontrar los análisis del sentimiento que están a la base de sus lecciones sobre la teoría del valor. Aquí aparecen descripciones minuciosas sobre la distinción entre cosa y valor, así como entre la apercepción y la motivación empírica y la afectiva; también analiza la fundamentación y el cumplimiento de los actos valorativos (a modo de analogía con la esfera judicativa), la relación de la alegría con el acto de valorar y las conexiones entre sentimientos, valores y juicios. Sin embargo, los estudios de Husserl en esta época no se limitan a la consideración del sentimiento como la estimación de un valor, sino que se internan en el análisis de la conciencia del sentimiento en su donación originaria como pura afección. Así, de entre los tipos de intencionalidad afectiva que Husserl encuentra, además de una 
intencionalidad que remite a un valor, aparece la intencionalidad de la afección del sentimiento (Gefühlsaffekts), los fenómenos de la expansión del sentimiento y los sentimientos co-estimulados, así como la pregunta central por la intencionalidad del temple de ánimo (Stimmung).

Antes de finalizar esta exposición, es de interés realizar una observación sobre los análisis de Husserl en los manuscritos de investigación que han dado lugar a los Studien acerca de este peculiar fenómeno que es el temple de ánimo y que ha despertado especial interés por determinados motivos ${ }^{21}$. El principal: su privilegiada conexión con todo lo que tiene que ver con el ámbito de las pasividades de la conciencia, ámbito en el que Husserl fue interesándose de manera creciente a lo largo de su trayectoria. Otro motivo es su proximidad con la «disposición afectiva» (Befindlichkeit) heideggeriana - a la que el pupilo de Husserl dio una importancia crucial en Ser y tiempo-, lo que permite establecer vínculos entre ambos conceptos. En la época previa a Ideas I, Husserl va a abordar el tema de la Stimmung desde al menos dos núcleos reflexivos distintos.

La aparición del temple de ánimo se sitúa en el contexto de una reflexión en la que Husserl compara el sentimiento que capta un valor y el sentimiento como disfrute, poniendo el ejemplo clarividente de que puedo encontrar algo alegre sin alegrarme ${ }^{22}$. Sin embargo, el sentimiento como disfrute no queda restringido a la categoría de mera sensación de sentimiento (placer, dolor...), sino que también va a poseer su propia intencionalidad. Y ahí va a jugar un rol determinante el temple de ánimo, que tiene la peculiaridad de ser una unidad de sentimiento que se propaga de objeto en objeto y de permanecer aun cuando el objeto motivador del sentimiento ya no esté presente. El temple de ánimo no es un sentimiento que se dirige a su objeto de valor, sino más bien es una unidad de sentimiento motivada a partir de diferentes valoraciones y reacciones de valor. En este sentido, Husserl establece la distinción básica entre el acto del sentimiento (la alegría por algo), la reacción del sentimiento (el vivir en esa alegría por algo) y la unidad del temple de ánimo (la unidad del sentimiento que se conserva en la corriente de la conciencia en su conjunto y que perdura

21 Sobre el tema del temple de ánimo, son muy enriquecedores los estudios que han realizado en México Antonio Zirión Quijano e Ignacio Quepons Ramírez. Los más acabados y completos se encuentran en Zirión QuiJANo, A.: "Colorations and moods in Husserl's "Studien zur Struktur des Bewusstseins" (with a final hint towards the coloring of life)», en The New Yearbook for Phenomenology and Phenomenological Philosophy, Parker, R. y Quepons, I. (eds.), vol. XVI, New York: Routledge, 2018, pp. 41-75; y en Quepons, I., «Horizonte y temple de ánimo en la fenomenología de Edmund Husserl», Dianoia, vol. LXI, núm. 76, 2016, pp. 83-112.

22 Merece la pena destacar la variedad de ejemplos que aparecen en estos manuscritos y que suelen sustraérsele al lector de la obra editada: el placer de fumar un puro, la contemplación de la carrera del caballo, la belleza de la mujer amada, el goce ante la obra de arte, el quitarse de encima una mosca... También el empleo de conceptos metafóricos como los valles profundos del sentimiento, las referencias a la poesía de Goethe, etc. 
en el tiempo, aun cuando haya desaparecido el «algo» motivante) $)^{23}$. Las tres posiciones presentarían intencionalidad.

El segundo núcleo reflexivo en torno al temple de ánimo va a aparecer en su discusión con Moritz Geiger a propósito de la conciencia de los sentimien$\operatorname{tos}^{24}$. En un artículo de $1911^{25}$, Geiger afirma que la observación analítica de los sentimientos no es posible, ya que no se pueden objetivar mientras se viven. El acto de reflexionar sobre el sentimiento convirtiéndolo en objeto hace que el sentimiento tal y como es en sí se pierda. En lo que supone una crítica a la facultad reflexiva en general como acceso originario a las cosas, Geiger hace ver que en el caso de los sentimientos la observación influye sobre el fenómeno, modificándolo esencialmente. La crítica lleva una carga de profundidad que obliga a Husserl a un gran esfuerzo argumentativo en una polémica que habría que analizar en toda su extensión y de la que aquí tan solo se puede apuntar un detalle $^{26}$. Por ejemplo, la diferenciación que Husserl establece entre dos formas de observación que Geiger no ha contemplado: la observación del sentimiento como objeto y la observación del sentimiento como dándose en el ahora que se vive, donde uno mismo es espectador de su propia vivencia, lo que legitimaría la posibilidad de la observación.

Estos son solo algunos momentos puntuales del caudal reflexivo a través del que se condujo Husserl en su abordaje de los sentimientos y cuyo estudio puede enriquecer notablemente la discusión fenomenológica actual. Como ya se ha advertido, el problema de los sentimientos está incrustado en el corazón de las estructuras de la conciencia y afecta esencialmente a la comprensión global de la fenomenología como cuestión de fondo. La exploración de Husserl dejó el tema abierto e inconcluso, dando la impresión de no resolver las numerosas inseguridades descriptivas a las que fue conducido ${ }^{27}$, algunas de las cuales se han presentado más arriba. Sin embargo, la naturaleza indeterminada del objeto de estudio y los pasos vacilantes con los que obliga a aproximarse a él pueden aportar también un ensanchamiento y una nueva apertura ahora que contamos con la perspectiva de décadas de estudio y reflexión fenomenológica. Lo que antes era un panorama indeciso e irresoluto torna, en estos momentos en los que disponemos de tantas innovadoras y originalísimas líneas de pensamiento abiertas a partir de la obra de Husserl a lo largo del siglo XX, en un suelo fértil y un manantial dinámico del que volver a nutrirse. Como en el caso

23 Esta distinción se encuentra en un manuscrito del Archivo Husserl bajo la signatura: A VI 12 II/72.

24 Manuscrito A VI 8 I/ 60-65.

25 Geiger, M., «Das Bewusstsein von Gefühlen», in A. Pfänder (Hrsg.), Münchener Philosophische Abhandlungen: Theodor Lipps zu seinem sechzigsten Geburtstag gewidmet von früheren Schülern, Leipzig: Barth, 1911, pp. 125-162.

26 Un análisis muy completo de esta discusión en: Quepons, I., «Atención y conciencia de los sentimientos: el temple de ánimo como trasfondo afectivo de la vida concreta», en Ápeiron. Estudios de Filosofía (Ejemplar dedicado a: Filosofía y fenomenología / coordinado por Roberto Ranz y Roberto Vivero Rodríguez), número 3, octubre de 2015, pp. 157-169.

27 Melle, U., o. c., pp. 98-99. 
de los grandes y auténticos filósofos que marcaron un hito en la historia del pensamiento, volver a Husserl es siempre sinónimo de fecundidad.

\section{BiBLIOGRAFÍA}

\section{Obras de Edmund Husserl}

Husserl, E. (1984). Logische Untersuchungen (Husserliana XIX/2). The Hague: Martinus Nijhoff.

- (1979). Aufsätze und Rezensionen (1890-1910) (Husserliana XXII). The Hague: Martinus Nijhoff.

- (1988). Vorlesungen über Ethik und Wertlehre (1908-1914) (Husserliana XXVIII). Dordrecht: Kluwer.

- (2004). Wahrnehmung und Aufmerksamkeit. Texte aus dem Nachlass (1893-1912) (Husserliana XXXVIII). Dordrecht: Springer.

- (2014). Grenzprobleme der Phänomenologie. Analysen des Unbewusstseins und der Instinkte. Metaphysik. Späte Ethik. Texte aus dem Nachlass (1908-1937) (Husserliana XLII). Dordrecht: Springer.

- (2013). Ideas relativas a una fenomenología pura y una filosofía fenomenológica. México D.F.: Fondo de Cultura Económica.

- «Gefühlsbewusstsein - Bewusstsein von Gefühlen. Gefühl als Akt und als Zustand», en Studien zur Struktur des Bewusstseins, ed. Zweites Band, Melle, U. y Vongehr, T. (eds.), pp. 78-101. (edición en progreso).

\section{Complementaria}

Brentano, F. (1935). Psicología. Traducción de José Gaos. Madrid: Revista de Occidente.

Cabrera, C., "Sobre la racionalidad de la esfera afectiva y su vínculo con la razón teórica en la ética de Husserl», Revista de Filosofía (Universidad de Buenos Aires), 39 (2014/1), pp. 73-96.

Costa, V., «Las emociones como condición del cuidado de sí y del aprendizaje», en $\mathrm{Fe}$ nomenología de la educación y la formación, Salamanca: Sígueme, 2018, pp. 125-173.

Crespo, M., «Fenomenología, sentimientos e identidad. La contribución de la fenomenología husserliana de los sentimientos a la cuestión de la identidad personal», Anales del Seminario de Historia de la Filosofía, vol. 33, número 2, 2016, pp. 605-617.

Fernández Beites, P., «Sobre el dolor y el mal», en El deber gozoso de filosofar: Homenaje a Miguel García-Baró, Salamanca: Sígueme, 2018, pp. 63-78.

Ferrer, U. y Sánchez-Migallón, S., La ética de Edmund Husserl, Sevilla: Thémata, 2018.

García-Baró, M (2012). Sentir y pensar la vida. Madrid: Trotta.

Geiger, M., «Das Bewusstsein von Gefühlen», in A. Pfänder (Hrsg.), Münchener Philosophische Abhandlungen: Theodor Lipps zu seinem sechzigsten Geburtstag gewidmet von früheren Schülern, Leipzig: Barth, 1911, pp. 125-162.

Gyemant, M., «Is feeling something knowing something? On the intentionality of feelings in Husserl's early writings (1894-1913)», en The New Yearbook for Phenomenology and Phenomenological Philosophy, Parker, R. y Quepons, I. (eds.), vol. XVI, New York: Routledge, 2018, pp. 87-108. 
Lee, N. I., «Edmund Husserl's Phenomenology of Mood», en Alterity and Facticity: New perspectives on Husserl, N. Depraz \& D. Zahavi (eds.), Drodrecht: Kluwer, 1998, pp. 102-120.

Melle, U., «Husserls deskriptive Erforschung der Gefühlserlebnisse», en Life, Subjectivity \& Art: Essays in Honor of Rudolf Bernet, Phaenomenologica 201, Breeur, R. y Melle, U. (eds.), Dordrecht: Springer, 2012, pp. 51-99.

Quepons, I., «Atención y conciencia de los sentimientos: el temple de ánimo como trasfondo afectivo de la vida concreta», en Ápeiron. Estudios de Filosofía (Ejemplar dedicado a: Filosofía y fenomenología / coordinado por Roberto Ranz y Roberto Vivero Rodríguez), número 3, octubre de 2015, pp. 157-169.

Quepons, I., «Horizonte y temple de ánimo en la fenomenología de Edmund Husserl», Dianoia, vol. LXI, número 76, 2016, pp. 83-112.

Rizo-Patrón de Lerner, R. (2015). El exilio del sujeto, Lima: Aula de Humanidades/Pontificia Universidad Católica del Perú.

Serrano de Haro, A., «Actos básicos y actos fundados. Exposición crítica de los primeros análisis husserlianos» en Anuario Filosófico, vol. 28, número 1, 1995, pp. 61-89.

Vigo Pacheco, A., «Intuición categorial», Thémata: Revista de filosofía, número 28, 2002, pp. 187-212.

Vongehr, T., «Husserl über Gemüt und Gefühl in den Studien zur Struktur des Bewusstseins», en B. Centi y G. Gigliotti (comps.), Fenomenologia della ragion pratica: l'etica di Edmund Husserl, Nápoles: Bibliopolis, 2004, pp. 227-253.

Zirión Quijano, A., "Colorations and moods in Husserl's "Studien zur Struktur des Bewusstseins" (with a final hint towards the coloring of life)", en The New Yearbook for Phenomenology and Phenomenological Philosophy, Parker, R. y Quepons, I. (eds.), vol. XVI, New York: Routledge, 2018, pp. 41-75.

Zirión Quijano, A., «El resplandor de la afectividad», Acta Fenomenológica Latinoamericana, vol. III, Lima/Morelia: CLAFEN/PUCP/UMSNH, 2009, 139-153.

CSIC. Instituto de Filosofía / Universidad Pontificia Comillas

Jesús Miguel Marcos Del Cano jesusmiguelmarcos@comillas.edu

[Artículo aprobado para publicación en junio de 2019] 
